\title{
Recognizing nurse stakeholder dissonance as a critical determinant of patient safety in new healthcare information technologies
}

\author{
Elizabeth A. Samaras ${ }^{*}$, Sara D. Real, Amber M. Curtis and Tessa S. Meunier \\ Department of Nursing, Colorado State University-Pueblo, 2200 Bonforte Blvd. Pueblo, Colorado, 81001 US
}

\begin{abstract}
Proper identification of all stakeholders and the comprehensive assessment of their evolving and often conflicting Needs, Wants, and Desires (NWDs) is a fundamental principle of human factors science and human-centered systems engineering; it is not yet a consistent element in development and deployment of new health information technologies (HIT). As the single largest group of healthcare professionals, nurses are critical stakeholders for these new technologies. Careful analysis can reveal nurse stakeholder dissonance (NSD) when integrating new technologies into the healthcare environment. Stakeholder dissonance is a term that describes the conflict between the NWDs of different stakeholders which, if left unresolved, can result in dissatisfaction, workarounds, errors, and threats to patient safety. Three case studies drawn from the authors' experience in a variety of acute-care settings where new HITs have been recently deployed are examined to illustrate the concept of NSD. Conflicting NWDs, other stakeholders, and possible root causes of the NSD are analyzed and mapped to threats to patient safety. Lessons learned, practical guidance for anticipating, identifying, and mitigating NSD, future research and implications for HFE and nursing practice are discussed.
\end{abstract}

Keywords: 'case studies', CPOE, EMR, 'human factors'

\section{Introduction, problem and purpose}

In the United States (US), as documentation requirements from regulatory and accrediting agencies such as the Centers for Medicare and Medicaid and The Joint Commission (TJC) have become increasingly more stringent, and the nation's healthcare facilities and providers are gearing up to demonstrate "meaningful use" under the HITECH incentive provisions of the 2009 ARRA Bill, there is a dramatic increase in the implementation of new Healthcare Information Technology (HIT) at all levels of the healthcare delivery system. Vendors are touting a plethora of computerized electronic health record, eprescribing, and ordering systems with the promise of streamlining older forms of documentation, improving communication, reducing errors, and in the process, "saving lives". Despite this climate of optimism and fervor, reports of adverse "unintended consequences" $[1,2,3]$ or "e-iatrogenesis" $[4]$ never- theless exist. As the single largest group of healthcare professionals [5], and principal patient advocates, nurses are critical stakeholders for these new technologies. Careful analysis can reveal nurse stakeholder dissonance (NSD), when integrating new technologies into the healthcare environment. Stakeholder dissonance is a term that describes the conflict between the Needs, Wants and Desires (NWDs) of different stakeholders, which if left unresolved, can result in dissatisfaction, workarounds, errors, and threats to patient safety [6].

The purpose of this paper is to analyze three case studies drawn from acute care settings to illustrate specific examples of Nurse Stakeholder Dissonance (NSD) that arose during the recent implementation of a new HIT. Lessons learned, practical guidance for anticipating, identifying, and mitigating NSD, as well as implications for patient safety, future research and practice is discussed.

\footnotetext{
* Corresponding author. E-mail: elizabeth.samaras@colostate-pueblo.edu; 719-549-2502
} 


\section{Methodology and theoretical development}

The concept of stakeholder dissonance (SD) is the framework for the current study, based upon earlier research conducted by Samaras and Samaras [7] and subsequent formal concept analysis [8], using Walker and Avant's approach [9]. Results of that analysis are beyond the scope of this short paper, but include that (a) SD is readily distinguished from Festinger, Riecken, and Schachter's cognitive dissonance (CD), [10] and (b) it describes disharmony among those having a stake or claim in the outcome of an endeavor, a collision of values between "any group or individual who can affect or is affected by the achievement of the organization's objectives" [11]. It is simply the conflict between the "Needs, Wants and Desires (NWDs)" of different stakeholders (where "Needs" = must have it; "Wants" = like to have it; "Desires" = I'll know it when I see it). If left unresolved, SD can result in dissatisfaction, workarounds, errors, and threats to patient safety [6].

Case studies were developed from the authors' personal experiences as Registered Nurses in the acute care settings described in each specific case study. Each case study was analyzed for sources of NSD and conflicting NWDs, other stakeholders, and possible root causes of the conflict (see Table 1). NSDs were then summarized and mapped to the threats to patient safety exposed in the three case studies (see Table 2).

\section{Case studies}

\subsection{Case one: post-operative unit and CPOE}

Case Study 1 occurred in a 36-bed post-operative, medical-surgical and orthopedic unit of a Regional Medical Center in the Southwest US. This inpatient acute-care unit deals with a range of diseases and problems such as small bowel obstructions, ventral hernia repairs, elective total joint procedures, and fractures resulting from falls and other traumatic events. There are currently approximately forty team members working on the unit (days and nights) which include registered nurses (RN's), patient care technicians (PCT's), health unit coordinators (HUC's), and team leaders (TL's). Team members' ages range from 20-70; this diverse age group contributes to a variable learning curve regarding the new Computerized Provider Order Entry (CPOE) system.
The unit CPOE ties in with barcode medication administration and electronic charting.

Interruptions between the HUCs, RNs, and physicians have become a major issue on this unit since the CPOE implementation. Currently, there are specific written orders that physicians use on pre-printed order sets and they do not correspond with the CPOE screens. These orders may or may not have pathways into the order entry system. For example, a physician may hand write or verbally order "Foley catheter to gravity drainage"; to find this in the CPOE, the non-physician entering the order (as physicians do not currently enter their own orders) has to locate the specific verbiage used in the system, which is "insert Foley". Because these two orders are not exactly the same, this type of "translation" leaves a lot of room for human error, medication errors, and misinterpretation of orders.

On the orthopedic side of this unit, pain control is a major patient-care issue. The unit introduced a new anesthetic device shortly after the implementation of the CPOE system, known as "On Q-Ball". This device provides continuous pain relief delivered by a $400 \mathrm{ml}$ ball of $2 \%$ Ropivacaine or a continuous flow of $5 \%$ Marcaine. It involves insertion of a small catheter, much like that of an epidural, into the groin, back, or rib area for pain relief, controlled by a dial that allows the medication to run from $2 \mathrm{ml} / \mathrm{hr}$ $14 \mathrm{ml} / \mathrm{hr}$. The amount of pain relief is determined by dose of medication "dialed", as well as the accuracy of anatomical placement to cover the affected nerves. Correct placement can also affect whether a patient can move their toes or dorsi-flex their feet, which nurses monitor. The Q-ball orders are managed by Anesthesiology, with about twenty different orders accompanying this type of pain management regimen. These companion orders include alternative pain management, if the anesthetic is ineffective in a given patient, management of "itchiness" and other side effects of the drugs, "select a flow" dosages, and the periodicity for required circulation, motor and sensation (CMS) checks . Many additional orders accompanying the Q-ball are among the most commonly missed or misinterpreted. Since the CPOE system does not contain "order sets", every single one of the orders comprising a "set" has to be entered by hand. What the prescriber writes on the paper is left to be "interpreted" by the person entering the orders, based upon available choices. While pharmacy enters the orders regarding the medications, the HUC, RN or TL is left to find the rest of the information within the CPOE system. Some of the staff is savvier with computer terminology and with finding information 
using the computerized terms, while others are less so regarding the HIT terminology. Because some of the orders do not have a pathway in the system, staff has resorted to typing up the orders and inserting them under a "miscellaneous" order category. Needless to say, this leaves the nursing and other staff routinely searching for the orders and during chart audits, as well; it increases the chance for order entry error and missed orders.

Another issue has arisen in transcribing the orders directly from the pre-printed order sets. It is often difficult to determine precisely what the prescriber wants with regards to the medication administration, resulting in numerous telephone calls placed to the physician for order clarification. One such example is found in the "select-a-flow" dosages. When the anesthesiologist writes $6 \mathrm{mls} / \mathrm{hr}$ on the order sheet, then $6 \mathrm{mls} / \mathrm{hr}$ is entered as such by the pharmacist, and is the dose available to the patient. However, many times the physician intended their order to be interpreted as a range, so that the dosage can be turned up or down as needed for pain relief. When the range is not explicitly entered electronically there is a gap in communication and no corresponding range is entered by pharmacy; this ultimately results in the potential for poor therapeutic pain management for the patient. Part of the problem in implementing pain control for total joint patients, which is often overlooked, is that these patient's need to have adequate movement and strength to participate in therapy as well as fluctuating requirements for pain relief. A single fixed dose does not provide sufficient flexibility to meet these evolving requirements. (See Table 1 for a summary of sources of NSD and conflicting Needs Wants and Desires (NWDs), other stakeholders ( $\mathrm{SH})$ involved and possible root causes for this case study.)

\subsection{Case two: trauma center implementing a CPOE}

Case Study 2 occurred in the special trauma room/suite in the operating room department for a Level II Regional Trauma Center in the US. This operating room is often used for trauma patients with multiple life-threatening injuries. This case involved a critically injured patient brought to the trauma suite for surgery to identify the source of bleeding and to help replace depleted blood volume. Due to the severity of bleeding, multiple units of blood product were needed. Unfortunately, sufficient blood product was not made available for administration due to CPOE-related problems and interagency barriers. Although the patient was severely injured and would likely have expired from these injuries, the cascade of failures, including problems with the new CPOE, may have contributed to this death.

Time is not a ready luxury when dealing with a patient that is losing blood volume faster than it can be replaced. In this situation, the trauma team was unable to effectively convey the urgency of the situation to the Blood Bank through the new CPOE as it was configured -- the system itself was limited and required staff to enter repeated single unit orders, rather than allowing for a single order for larger amounts of blood product at one time. Months earlier, a massive transfusion order could have been given verbally, but now with the introduction of the CPOE, the Blood Bank required that all orders had to be sent electronically. In this emergency, computer orders for more units of blood could not be entered quickly enough to stay on top of the patient's need for large quantities of blood products, thus resulting in a limited (and inadequate) supply of blood products available for infusion into the patient. Although the Blood Bank was functioning within their established policies and procedures, which did not allow them to release blood products without an electronically-generated physician order, there was no mechanism in place to order the large quantities required for this patient beyond issuing constantly repeated single unit order requests for blood products. This ordering hurdle proved to be insurmountable and too time consuming for the trauma team, who were simultaneously dealing with the other emergency care needs of this critically-injured and hemorrhaging trauma patient. Calls made to the blood bank describing the criticality of the situation and demanding blood products were responded with, "We will issue blood products when we have received appropriate physician order entry." The trauma team staff involved in resuscitation effort felt helpless as they were unable to access the necessary lifesaving resources for this patient. (Refer to Table 1, Case Study 2 for further analysis). 
Table 1

Summary of sources of NSD/conflicting NWDs, other stakeholders (SH), \& possible root causes by case study

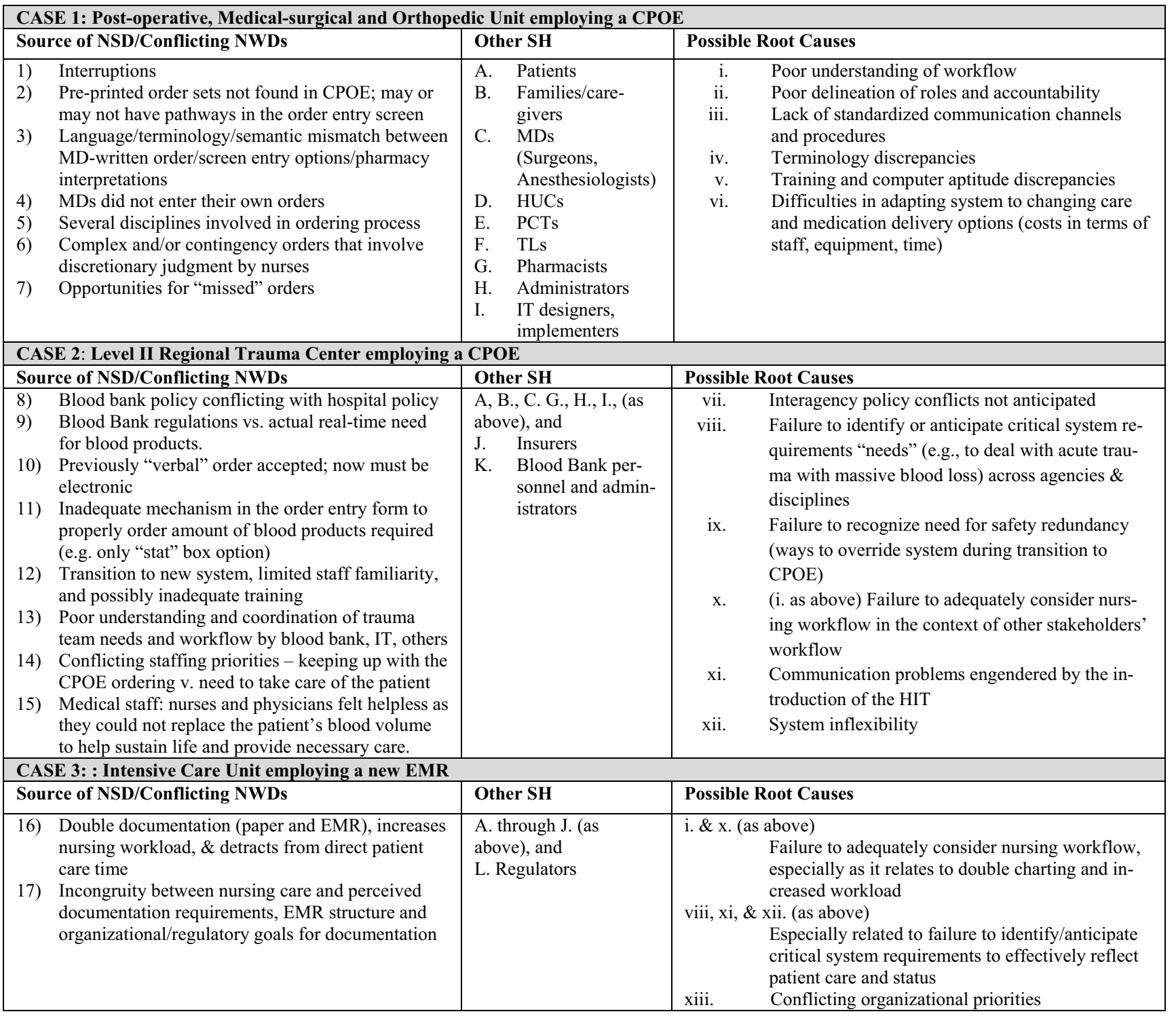

\subsection{Case three: intensive care unit and an EMR}

The setting for the third case study is a 38 bed Intensive Care Unit (ICU) located in an urban Level II trauma center in Northern Colorado where the introduction of the Electronic Medical Record (EMR) is creating dissonance among the nursing staff stakeholders. In this ICU, the EMR is used throughout the course of the patient's stay. A patient's admission history and assessment is recorded through the EMR. In addition, medication administration, treatments, education, and discharge planning are all recorded in the EMR as required by regulatory agencies. While the EMR is recognized to be beneficial to organizational goals, its use is perceived by nurses on this unit to be in direct conflict with the caring, humanistic perspective that defines nursing practice. Nurse stakeholder dissonance in this setting has evolved as the EMR has become increasingly utilized in patient care. Nurses on this unit are required to document on a paper record, as well as in the EMR. This imposition to spend increased time for dual documentation 
is perceived by nurses as diverting attention from their more important focus on direct patient care.

Additionally, nurses are required to quantify and categorize the care they provide based on predetermined fields in the EMR. For example, it is a TJC requirement to document a pain assessment on a patient. There is currently no option in the EMR to note if the patient is sleeping. Nurses are then faced with a decision: 1) assume the patient has no pain, because they are sleeping, 2) wake the patient to ask about their pain level, or 3) skip the documentation entirely. Each one of these options is likely to cause a conflict for the nurse, whose first concern is providing patient-centered care. These issues have resulted in "workarounds" and inaccurate documentation in order to remain compliant with documentation requirements. In addition to nurse dissatisfaction, there is potential for dissonance from patients, managers, physicians, and regulatory agencies due to these inaccuracies and threats to safety that may emerge as a result of these maneuvers.

Table 2

Summary of identified Nurse Stakeholder Dissonance (NSD) mapped to threats to patient safety

\begin{tabular}{|c|c|}
\hline Nurse Stakeholder Dissonance & Threats To Patient Safety (physical, psychological, and financial safety) \\
\hline $\begin{array}{l}\text { NSD Related to Nursing Values of Caring and Patient Advo- } \\
\text { cacy } \\
\text { Nursing practice is based upon caring; advocacy is a fundamental } \\
\text { principle of the nursing code of ethics [12]. } \\
\text { In these case studies, missed orders, incorrect orders and dosag- } \\
\text { es, poor pain management, feelings of helplessness, and inade- } \\
\text { quate means for ordering needed blood supplies threaten these } \\
\text { core nursing values and roles. }\end{array}$ & $\begin{array}{l}\text { Case 1) Misinterpretation of order: } \\
\rightarrow \text { Potential for error and threat to patient safety } \\
\rightarrow \text { Poor pain management } \\
\rightarrow \text { Reduction in quality care and patient satisfaction may also result in } \\
\text { financial impacts to patient related to incorrect orders } \\
\text { Case 2) Problems with the HIT implementation: } \\
\rightarrow \text { Resultant failure to procure adequate blood supplies } \\
\rightarrow \text { Contributed to an unacceptable patient outcome - death. }\end{array}$ \\
\hline $\begin{array}{l}\text { NSD Related to the Domain and Scope of Practice of the } \\
\text { Nurse } \\
\text { When nurses are pushed beyond their scope of practice, judgment } \\
\text { may be compromised that may result in threats to patient safety. } \\
\text { Similarly, when nurses are unable to utilize their full capabilities } \\
\text { and professional judgment, they may not practice according to } \\
\text { their level of practice or accepted standards of care [13]. } \\
\text { In the case studies, this was demonstrated by failures in commu- } \\
\text { nication, in procuring needed supplies, in adequately considering } \\
\text { nursing practice and workflow, as well as in general inflexibility } \\
\text { of systems to meet changing requirements. }\end{array}$ & $\begin{array}{l}\text { Case 1) Over or under reliance on nursing judgment or discretion as a } \\
\text { result of poorly delineated orders - may result in threat to patient safety } \\
\text { and jeopardize nurses' licensure } \\
\text { Case 2) Nurse may be unable to provide adequate care to standard of prac- } \\
\text { tice } \\
\text { Case 3) Nurses may be unable to effectively document their care according } \\
\text { to their professional judgment and standards; this may result in poorer } \\
\text { patient communication and resultant threats to patient safety }\end{array}$ \\
\hline $\begin{array}{l}\text { NSD Related to the Provision of Safe, Efficient, Effective, } \\
\text { Patient-Centered Care } \\
\text { Providing safe, efficient, effective, evidence-based, patient- } \\
\text { centered care are central hallmarks of the Institute of Medicine's } \\
\text { agenda for quality healthcare [14]and provider education [15] }\end{array}$ & $\begin{array}{l}\text { Case 1) Misinterpretation and potential for missed order; need for nu- } \\
\text { merous calls for order clarifications wastes time and fails to optimally } \\
\text { meet patient needs; interruptions divert attention away from patient care } \\
\text { Case 2) Conflicting RN priorities - keeping up with the institutionally- } \\
\text { required CPOE ordering v. need to take care of the patient; inability to } \\
\text { provide efficient and effective care, resulting in poorer patient outcomes } \\
\text { Case 3) Double documentation (paper and EMR): } \\
\rightarrow \text { Increases nursing workload } \\
\rightarrow \text { Detracts from direct patient care time } \\
\text { Case 3) Incongruity between nursing care and perceived documentation } \\
\text { requirements, EMR structure and organizational/regulatory goals for do- } \\
\text { cumentation: } \\
\rightarrow \text { Nursing workarounds } \\
\rightarrow \text { Inaccurate documentation may result in errors } \\
\rightarrow \text { Widespread dissatisfaction may threaten patient safety and care }\end{array}$ \\
\hline
\end{tabular}


In the sleeping patient example above, the patient's pain may not be adequately addressed. This can result in patient dissatisfaction, delayed healing, and emotional harm. There is also the potential for adverse organizational ramifications, including poor Hospital Consumer Assessment of Health Providers and Systems (HCAHPS) scores and even lawsuits. Problems with the EMR may be rooted in a failure to adequately consider nursing workflow. Current practice on this unit requires both paper and EMR charting. This dual charting system increases the workload for nurses, as well as increasing time constraints resulting in workaround practices and less time for patientcentered care. Additionally, there is incongruity between the EMR documentation and nursing documentation. EMR documentation is often focused on achieving organizational goals such as the Surgical Care Improvement Project (SCIP) and National Patient Safety Goals (NPSG), while nursing documentation is aimed at patient-centered care. This discrepancy between objectives can create a new dichotomy that requires further consideration. (Refer to Table 1, Case Study 3 for analysis).

\section{Critical evaluation}

Review of the three case studies demonstrates that common themes of NSD begin to emerge from the analysis of individual sources of NSD and conflicting NWDs (see Table 1). Conflicting NWDs are found related to: a) nursing values of caring and patient advocacy, b) the domain and scope of nursing practice, and c) the provision of efficient, effective, patient-centered care (See Table 2). Furthermore, we see threats to patient safety (emotional, physical, and even financial safety) that are directly related and mapped to these NSD themes in Table 2.

\subsection{Lessons learned}

In the Case 1 facility, actions have been taken to address the problems identified in the case study. The need for orthopedic surgeons and the anesthesiologists to collaborate on flexible pain management protocols that allow the nurse to use professional judgment to adjust "select-a-flow" medication dosages within appropriate ranges based upon the patient's CMS assessment and pain rating has been recognized. These protocols must then be reflected precisely in the CPOE system to ensure correct communication and follow-through. The hospital now holds focus groups (including IT, physicians, RN's and others responsible for order entry), so that every discipline is on the same page before the introduction of new treatments and procedures. By the middle of 2012, prescribers will be responsible for entering their orders directly into system. This should minimize misinterpretation of orders; it will not resolve problems identified with direct provider order entry.

The Case 2 setting has made a number of changes as a result of this incident. A Massive Transfusion Protocol was developed for critically hemorrhaging and potentially salvageable patients. This protocol only requires two orders to be entered: one to start the massive delivery of blood products, and the other to stop it. Upon entering the order, an automatic 10 units of red blood cells would be delivered and the Blood Bank will endeavor to "stay head" with multiple units of blood products at the ready (e.g. units of fresh frozen plasma, cryoprecipitate and platelet packs). This protocol also requires the Blood Bank to make hourly phone calls to the operating room, fostering open communication and monitoring of the patients' progress.

In the ICU setting from case study 3, nurses are still performing dual charting and still finding themselves frustrated with an increased workload that diverts them from patient care. The situation remains fraught with NSD.

\subsection{Conclusions and recommendations}

By examining three illustrative case studies, sources of NSD have been identified, including the large contribution played by conflicts between nurses and other stakeholder NWDs, during the introduction of new HIT. Ideally, a priori and iterative assessment of all stakeholder NWDs during the design and deployment phases of the new technologies would facilitate identification and mitigation of SD before problems occur and lives are lost [16]. As shown in this analysis, NSD can be directly linked to significant threats to patient safety; recognizing and resolving NSD warrants particular attention both before HIT deployment and during its useful lifetime. More research into the importance of NSD as a factor in the complex socio-technical domain of HIT implementation is recommended. 


\section{References}

[1] Koppel R, Metlay JP, Cohen A, Abaluck B, Localio AR, Kimmel SE, et al. Role of computerized physician order entry systems in facilitating medication errors. JAMA. 2005; 293 (10):1197-1203.

[2] Ash JS, Sittig DF, Dykstra RH, Guappone K, Carpenter JD, Seshadri V. Categorizing the unintended sociotechnical consequences of computerized provider order entry. International Journal of Medical Informatics. 2007 June; 76 supplement 1:S21-S27.

[3] Bernstam EV, Hersh WR, Sim I, Eichmann D, Silverstein JC, Smith JW, et al. Unintended consequences of health information technology: A need for biomedical informatics. JBI. 2010, Oct; 43 (5): 828-830.

[4] Weiner JP, Kfuri $\mathrm{T}$, Chan $\mathrm{K}$ and Fowles JB. "e-Iatrogenesis": the most critical unintended consequence of CPOE and other HIT. J Am Med Inform Assoc. 2007 ; 14: $387-388$ as cited by Bernstam, et al., 2010

[5] U.S. Department of Health and Human Services, Health Resources Services Administration. Initial Findings from the 2008 National Sample Survey of Registered Nurses.2010.Available from: http://bhpr.hrsa.gov/healthworkforce/rnsurveys/rnsurve yinitial2008.pdf

[6] Samaras EA, Samaras GM. Using Human-Centered Systems Engineering to Reduce Nurse Stakeholder Dissonance. Biomedical Instrumentation \& Technology. 2010 Nov; 44(s1):25-32.

[7] Samaras GM, Samaras EA. Feasibility of an e-health initiative: Information NWDs of cancer survivor stakeholders. Proceedings of International Ergonomics Association World Congress; 2009: Beijing, China: International Ergonomics Association.

[8] Samaras EA. A concept analysis of stakeholder dissonance and implications for nursing practice. Unpublished. 2011.
[9] Walker LO, Avant KC. Strategies for theory construction in nursing. 5th ed. Boston: Prentice Hall; 2011.

[10] Festinger, L, Riecken, HW, Schachter S. When prophecy fails. Minneapolis: University of Minnesota Press; 1954

[11] Freeman, RE. Strategic management: A stakeholder approach. Boston: Pitman; 1984 as cited by Mitchell RK, Agle BR, Wood DJ. Toward a theory of stakeholder identification and salience: Defining the principle of who and what really counts. Academy of Management Review.1997; 22(4):853. Available from EBSCOhost.

[12] American Nurses Association. Code of Ethics for Nurses with Interpretive Statements. 2004. Available from http://www.nursingworld.org/codeofethics.

[13] American Nurses Association. Nursing: Scope and Standards of Practice. 2004. Silver Spring, MD: Author.

[14] Institute of Medicine. Crossing the quality chasm: A new heath system for the 21 st century. 2001. Available from

http://books.nap.edu/openbook.php?record id=10027.

[15] Institute of Medicine. Health Professions Education: A Bridge to Quality. 2003. Available from: http://www.iom.edu/Reports/2003/Health-ProfessionsEducation-A-Bridge-to-Quality.aspx

[16] Samaras, GM. Human-centered systems engineering: Managing dissonance in healthcare delivery. In Kolker A, Story P, editors. Management engineering for effective healthcare delivery: Principles and practices. Philadelphia: IGI Global; 2011. 148-171. 